\title{
A New Image Registration Method Robust to Noise
}

\author{
Guangyi Chen and Stéphane Coulombe \\ Department of Software and IT Engineering, École de technologie supérieure, Université du Québec, 1100 Notre-Dame \\ West, Montréal, Québec, Canada H3C 1K3. \\ Email: \{guangyi_chen@hotmail.com, Stephane.Coulombe@etsmtl.ca\}
}

\begin{abstract}
Image registration is a fundamental task in many image processing applications. In this paper, we estimate the translation, rotation, and scaling parameters between a reference image and a distorted image in order to register them. We use the ratio of means to estimate the scaling factor, the Radon transform to estimate the rotation angle, and the fast Fourier transform (FFT) to estimate global spatial shifts. Experimental results show that our proposed method can estimate the transformation parameters very accurately. Furthermore, our method performs very well in noisy environments.
\end{abstract}

Keywords: Translation-invariant; rotation-invariant; scaleinvariant; image registration; Radon transform; FFT.

\section{Introduction}

Image registration is a very important task in applications such as medical imaging, automatic target recognition, image quality assessment, etc. Keller et al. [1] used pseudo-polar-based estimation of large translations, rotations, and scalings in images. Wolberg and Zokai [2] also worked on robust image registration by using the logpolar transform. Varghese and Wang [3] used the Fourier transform to estimate global spatial shifts. Reddy and Chatterji [4] proposed an FFT-based technique for translation, rotation and scale-invariant image registration. Jafari-Khouzani and Soltanian-Zadeh [5] applied the Radon transform to orientation estimation for rotation invariant texture analysis. De Castro and Morandi [6] developed an image registration method for translated and rotated images using finite Fourier transforms. However, this method fails in the presence of scale change. Wei et al. [7] estimated the image rotation angle by using interpolation-related spectral signatures with application to blind detection of image forgery. Lowe [8] proposed a distinctive image feature approach for scale-invariant key point extraction and registration (SIFT), and Bay et al. [9] developed the SURF: Speeded Up Robust Feature, which was claimed to be faster than SIFT.

In this paper, we propose a new image registration method. We use the ratio of means to estimate the scaling factor between the reference and distorted images, the Radon transform to estimate the rotation angle between the two images, and the 2D FFT to estimate the global spatial shifts (translation). Experimental results show that our proposed method performs very well for the tested images and with the LIVE image quality assessment database release 2 [11]. In addition, it works very well in noisy environments.

The rest of this paper is organized as follows. Section II proposes a new method for estimating the translation, rotation, and scaling parameters between the reference image and the distorted image in order to register them. Section III conducts certain experiments in order to show the advantages of the proposed method over existing methods appearing in the literature. Finally, Section IV draws the conclusions of this paper.

\section{Proposed Method}

In this paper, we propose a new image registration method by means of the Radon transform and the FFT. Let image $Y$ be the rotated, and scaled version of image $X$. If $X$ and $Y$ do not have the same number of pixels, then we pad zeros around the smaller image. The Fourier transform of images $X$ and $Y$, each of size $M \times N$, are:

$$
\begin{aligned}
& F_{1}(u, v)=\frac{1}{M N} \sum_{m=1}^{M} \sum_{n=1}^{N} X(m, n) e^{-i(u m / M+v n / N)} \\
& F_{2}(u, v)=\frac{1}{M N} \sum_{m=1}^{M} \sum_{n=1}^{N} Y(m, n) e^{-i(u m / M+v n / N)} .
\end{aligned}
$$

In the polar coordinate system, their magnitudes are related as (see [4] for details):

$$
\left\|F_{1}(r, \theta)\right\|=\frac{1}{a^{2}}\left\|F_{2}\left(r / a, \theta-\theta_{0}\right)\right\|
$$

where $a$ is the scaling factor (horizontally and vertically) and $\theta_{0}$ is the rotation angle between the two images, and

$$
\begin{gathered}
r=\sqrt{m^{2}+n^{2}} \\
\theta=\tan ^{-1}(n / m) .
\end{gathered}
$$

Therefore, we can obtain the scaling factor: 


$$
a=\sqrt{\frac{\left\|F_{2}(0,0)\right\|}{\left\|F_{1}(0,0)\right\|}} .
$$

We can obtain the scaling factor $a$ without computing the 2D FFT of images $X$ and $Y$, since

$$
F_{1}(0,0)=\frac{1}{M N} \sum_{m=1}^{M} \sum_{n=1}^{N} X(m, n)
$$

and

$$
F_{2}(0,0)=\frac{1}{M N} \sum_{m=1}^{M} \sum_{n=1}^{N} Y(m, n)
$$

Now, consider that $Y$ is translated as well as rotated and scaled. The approach used to compute the scaling factor $a$ will still hold. We create a mask image: $Y_{0}(m, n)=1$ if $Y(m, n)>\tau$; and $Y_{0}(m, n)=0$, otherwise. In this paper, we have chosen $\tau=40$. We calculate the centroid $\left(\mathrm{c}_{\mathrm{r}}, \mathrm{c}_{\mathrm{c}}\right)$ of $Y_{0}$ as:

$$
\begin{aligned}
& c_{r}=\sum_{m, n} m Y_{0}(m, n) / \sum_{m, n} Y_{0}(m, n) \\
& c_{c}=\sum_{m, n} n Y_{0}(m, n) / \sum_{m, n} Y_{0}(m, n)
\end{aligned}
$$

We move $Y\left(c_{r}, c_{c}\right)$ to the center of image $Y$ and denote the new image as:

$$
Y_{1}(m, n)=Y\left(m-c_{r}+M / 2, n-c_{c}+N / 2\right) .
$$

where $\quad m \in[1, M] \quad$ and $\quad n \in[1, N] . \quad$ If $\left(m-c_{r}+M / 2, n-c_{c}+N / 2\right)$ is outside the index range $[1, M] \times[1, N]$, we set $Y_{1}(m, n)=0$. We can then use the calculated scaling factor $a$ to resize the image $Y_{l}$ such that it has the same scale as the image $X$ in order to estimate the rotation angle. Let us denote it as $Y_{2}(m, n)=Y_{1}(m / a, n / a)$.

The rotation angle $\theta_{0}$ between the reference image $X$ and the normalized image $Y_{2}$ can be obtained using the Radon transform. The Radon transform of a 2D discrete function $A(x, y)$ is defined as [14]:

$$
R(r, \theta)=\iint_{x} A(x, y) \delta(r-x \cos \theta-y \sin \theta) d x d y
$$

where $\delta()$ is the Dirac delta function. As pointed in [14], for modern applications it is important to have a discrete 2D Radon transform, which has been the object of attention of many authors over the last twenty years. Until recently, the Radon transform lacked a coherent discrete definition for 2D discrete images, which is algebraically exact, invertible, and rapidly computable. Therefore, the authors of [14] defined a notion of 2D discrete Radon transforms for discrete $2 \mathrm{D}$ images, which is based on summations along lines of absolute slope less than 1. Values at non-grid locations are defined using trigonometric interpolation on a zero-padded grid. They proved that their definition provides a faithful description of the continuum, as it converges to the continuous Radon transform as the discretization step approaches zero. More details about the discrete Radon transform can be found in [14]. According to [14], we can perform the discrete Radon transform of $\mathrm{X}$ and $\mathrm{Y} 2$, denoted as $R_{1}(r, \theta)$ and $R_{2}(r, \theta)$, respectively, where both of them are of size $\mathrm{K} \times \mathrm{L}$. We know that $R_{1}(r, \theta)$ and $R_{2}(r, \theta)$ are the same, except for the circular shift along the $\theta$ direction, i.e., $R_{2}(r, \theta)=R_{1}\left(r, \theta+\theta_{0}\right)$. We can use the circular crosscorrelation to calculate $\theta_{0}$, but it is time-consuming, with a computational complexity of $O\left(K L^{2}\right)$. This is because for every row of $R_{1}$ and $R_{2}$, we need to circularly shift $R_{2}$ and then calculate the cross-correlation between the two rows. This has a computational complexity of $O\left(L^{2}\right)$. Since we have $K$ rows in total, the total computational complexity is $O\left(K L^{2}\right)$.

We briefly state the definition of the cross-correlation and its fast implementation here. The cross-correlation between two discrete real-valued functions of $f[n]$ and $g[n]$ is [12]:

$$
(f * g)[n]=\sum_{m=-\infty}^{\infty} f[m] g[n+m] .
$$

The convolution between $f[n]$ and $g[n]$ is [13]:

$$
(f \oplus g)[n]=\sum_{m=-\infty}^{\infty} f[m] g[n-m] .
$$

The cross-correlation of function $f[n]$ and $g[n]$ is equivalent to the convolution of $h[n]=f[-n])$ and $g[n]$ :

$$
(f * g)[n]=\sum_{m=-\infty}^{\infty} f[-m] \quad g[n-m]=(h \oplus g)[n] .
$$

Analogous to the convolution theorem, the cross-correlation satisfies:

$$
\begin{aligned}
F F T \quad\{f * g\} & =F F T \quad\{h \oplus g\} \\
& =F F T \quad\{h\} \bullet F F T\{g\} \\
& =\operatorname{conj}(F F T(f)) \bullet F F T \quad\{g\}
\end{aligned}
$$

where FFT denotes the fast Fourier transform, conj the complex conjugate, and the dot means component-wise 
multiplication. By taking the inverse $F F T$, we can obtain a fast implementation of $f * g$.

Based on the above discussion, we use the fast crosscorrelation to find $\theta_{0}$, which has a computational complexity of $O(K L \log L)$. Let us denote the $\mathrm{m}^{\text {th }}$ row of $R_{l}$ and $R_{2}$ as $r_{l}(n)$ and $r_{2}(n)$, respectively, where $n \in[1, L]$. We are looking for the offset for which the cross-correlation is maximum, i.e. for which the two rows $r_{l}(n)$ and $r_{2}(n)$ match the most. We perform 1D forward FFT on $r_{1}(n)$ and $r_{2}(n)$, and calculate $r_{3}(u)=\operatorname{conj}\left(F F T\left(r_{1}\right)\right) \bullet F F T\left(r_{2}\right)$, where . is the component-wise product. We then apply the 1D inverse $F F T$ to $r_{3}(u)$, i.e., $r_{4}(n)=\operatorname{IFFT}\left(r_{3}(u)\right)$. We find the location index $\theta^{*}(m)$ of the maximum value on every row of the obtained $r_{4}(n)$,i.e., $\theta^{*}(m)=-\max _{1 \leq n \leq L} r_{4}(n)$. We take the median of these maximum values as $\theta_{0}=$ median $\left(\theta^{*}(m)\right)$. We can then rotate the image $Y_{I}$ for $-\theta_{0}$ degrees in order to compensate for the orientation difference.

For translation, we choose the global motion compensation (MC) method proposed in [3], which is a simple, fast, and reliable method, providing integer pixel precision. Let $Y_{3}(m, n)$ be the image that has already been compensated for scaling and rotation, and $X(m, n)$ the reference image. Also,

$$
\begin{aligned}
& F_{1}(u, v)=\frac{1}{M N} \sum_{m=1}^{M} \sum_{n=1}^{N} X(m, n) e^{-i(u m / M+v n / N)} \\
& F_{2}(u, v)=\frac{1}{M N} \sum_{m=1}^{M} \sum_{n=1}^{N} Y_{3}(m, n) e^{-i(u m / M+v n / N)} .
\end{aligned}
$$

Then, we can define the fast cross-correlation function as:

$$
k_{F C}(m, n)=I F F T_{2}\left(\operatorname{conj} \quad\left(F_{1}(u, v)\right) \bullet F_{2}(u, v)\right),
$$

where $I F F T_{2}$ is the inverse 2D Fourier transform, ${ }^{c o n j}$ the complex conjugate, - the component-wise product. The estimated motion vector is given as:

$$
\left(m_{\text {opt }}, n_{\text {opt }}\right)=\underset{(m, n)}{\arg \max } k_{F C}(m, n) .
$$

Figs. 1-3 show the original image, its distorted version, and the images registered by the proposed method, SURF, and SIFT with noise standard deviation $\sigma_{\mathrm{n}}=10,50$ and 100 , respectively. It can be seen that at $\sigma_{\mathrm{n}}=100$, both SURF and
SIFT fail, but our proposed method works very well in this case. In addition, with scaling factor $\mathrm{a}=0.5$, all three methods generate good registration (see Fig. 4). However, with scaling factor $a=0.1$, both SURF and SIFT fail, whereas our proposed method works very well (see Fig. 5).

Noise is an undesirable phenomenon occuring during image capture that randomly varies the luminance or color intensity of its pixels. In this paper, we only consider Gaussian white noise because it is among the few most commonly occurring types of noise, and we leave other types of noise to our future research. For Gaussian white noise, its power within a given bandwidth is independent of frequency, where the probability of a particular amplitude follows a Gaussian distribution. Salt-and-pepper noise has dark pixels in bright regions and bright pixels in dark regions. Shot noise has a root-mean-square value proportional to the square root of the image intensity, and the noise at different pixels are independent of one another. Shot noise has a Poisson distribution, which is usually similar to Gaussian distribution. Quantization noise has an approximately uniform distribution and it is signal independent if other noise sources are big. Other types of noise include atmospheric noise, background noise, Brownian noise, burst noise, cosmic noise, flicker noise, grey noise, jitter, Johnson-Nyquist noise, and pink noise.

We only distort the second image by adding Gaussian white noise. If both images are corrupted by Gaussian white noise, then the estimated parameters between the two images will remain the same. This is due to the fact that Gaussian white noise has zero mean and the Radon transform is robust to this kind of noise. In addition, the spatial shifts between the two images are robust to noise as well [3].

The computational complexity of the proposed method in this paper is compared with two existing methods. In paper [4], the authors propose a technique to estimate translation, rotation, and scaling parameters. Their method uses 6 forward 2D FFT and 6 inverse 2D FFT, which is time-consuming and error-prone. In this paper, we use the ratio of means to estimate the scaling factor, and the Radon transform to estimate the rotation angle between the reference and distorted images. The Radon transform of an image can be carried out with constant time $O(1)$ on a reconfigurable mesh [10], or at most, at a complexity of a 2D FFT [5]. We then utilize the 2D FFT to obtain global spatial shifts [3]. Our method will use only 3 forward 2D FFT, 2 inverse 2D FFT and 2 Radon transforms. Therefore, we can conclude that our proposed method should be faster than the technique given in [4]. In [6], the authors propose an image registration method, which requires 180 inverse 2D FFT. Also, their method fails if a scale change is present. Conversely, our proposed method works well in the presence of scale changes, and its computational complexity is much less than that of [6]. 
The following are the major advantages of our proposed method in this paper over existing methods. Our method performs very well in noisy environments, whereas existing methods such as [8] and [9] will fail if the noise level is too high. In addition, our method is fast in terms of computational complexity $O(M N \log (M N)+K L \log (L))$, where the input images are of size $M \times N$ and the Radon image is of size $K \times L$. Furthermore, our method can estimate all three parameters (translation, rotation, and scaling), whereas most existing methods only calculate one or two parameters [5], [7]. Experimental results conducted in the next section show that our proposed method is feasible in registering images, especially in noisy environments.

\section{Experimental Results}

We performed some experiments on $512 \times 512$ Barbara and Lena images, and used the LIVE Image Quality Assessment Database Release 2 [11], which consists of 779 distorted images derived from 29 original images using five types of distortions. The distortions include JPEG compression, JPEG2000 compression, Gaussian white noise (GWN), Gaussian blurring (GBlur), and the Rayleigh fast fading (FF) channel model. We performed experiments on all 779 distorted images in the LIVE image database. We used Matlab code for our proposed method in this paper.

Tables 1 and 2 give the experimental results for the Lena and Barbara images with translation, rotation, and scaling distortion, and with added noise. In both tables, our estimated parameters are very close to the input distortion parameters that produced the distorted images. In addition, our proposed method performs very well in noisy environments, whereas at $\sigma_{\mathrm{n}}=100$, SIFT fails to find matching key points and SURF does not generate accurate results for the Lena image.

It should be pointed out that the precision of the rotation angle in the proposed method depends on the number of points $L$. In our simulations, we have used $L=180$ sample points in the rotation angle of the Radon transform. Therefore the precision is 1 degree (equivalent to 0.0174 radian). We can increase the accuracy by increasing the number of sample points, but this will increase the computational complexity. Therefore, there is a trade-off to make between parameter accuracy and computational complexity.

Table 3 tabulates the results when every distorted image in the LIVE database is further distorted by scaling factor 0.9 , rotation angle $0.1 \times 180 / \pi$ degrees, spatial shifts 4 pixels in the horizontal direction, and 4 pixels in the vertical direction, and Gaussin white noise. We take the mean of all estimated parameters as an estimated result for translation, rotation angle, and scaling factor. For this database, all three (the proposed, SIFT, and SURF) methods allow a good estimation of parameters when there is no noise added. Our proposed method performs better than SIFT and SURF when we add noise to the images in this database as shown in Table 3. For instance, for $\sigma_{\mathrm{n}}=50$, the SURF method estimates the translation parameters to be $(9,-5)$ while they should be $(4,4)$. It also gives a totally wrong estimate of the scale parameter. For $\sigma_{\mathrm{n}}=100$, SURF's results are even worst and can't estimate any of the parameters properly. For $\sigma_{\mathrm{n}}=100$, the SIFT method has a larger estimation error for translation and rotation than the proposed method.

Table 1. Matching results of a translated $(4,4)$, rotated $(0.1$ radian), and scaled (0.9) Lena image with different noise levels

\begin{tabular}{|c|c|c|c|c|}
\hline $\begin{array}{c}\text { Noise } \\
\text { level }\end{array}$ & Method & Translation & Rotation & Scale \\
\cline { 2 - 5 } & Original & $\mathbf{( 4 , 4 )}$ & $\mathbf{0 . 1 0 0 0}$ & $\mathbf{0 . 9 0 0 0}$ \\
\hline \multirow{3}{*}{$\sigma_{\mathrm{n}}=0$} & Proposed & $(4,4)$ & 0.0873 & 0.8983 \\
\cline { 2 - 5 } & SIFT & $(4,4)$ & 0.1001 & 0.9000 \\
\cline { 2 - 5 } & SURF & $(5,-5)$ & 0.0979 & 0.9076 \\
\hline \multirow{3}{*}{$\sigma_{\mathrm{n}}=50$} & Proposed & $(4,4)$ & 0.0873 & 0.8982 \\
\cline { 2 - 5 } & SIFT & Fail & Fail & Fail \\
\cline { 2 - 5 } & SURF & $(4,5)$ & 0.1091 & 0.9081 \\
\hline \multirow{3}{*}{$\sigma_{\mathrm{n}}=100$} & Proposed & $(4,4)$ & 0.0873 & 0.8981 \\
\cline { 2 - 5 } & SIFT & Fail & Fail & Fail \\
\cline { 2 - 5 } & SURF & $(27,20)$ & 0.5684 & 1.8512 \\
\hline
\end{tabular}

Table 2. Matching results of a translated $(4,4)$, rotated $(0.1$ radian), and scaled (0.9) Barbara image with different noise levels

\begin{tabular}{|c|c|c|c|c|}
\hline \multirow{2}{*}{$\begin{array}{c}\text { Noise } \\
\text { level }\end{array}$} & Method & Translation & Rotation & Scale \\
\cline { 2 - 5 } & Original & $\mathbf{( 4 , 4 )}$ & $\mathbf{0 . 1 0 0 0}$ & $\mathbf{0 . 9 0 0 0}$ \\
\hline \multirow{3}{*}{$\sigma_{\mathrm{n}}=0$} & Proposed & $(4,4)$ & 0.0873 & 0.8983 \\
\cline { 2 - 5 } & SIFT & $(4,4)$ & 0.0998 & 0.9002 \\
\cline { 2 - 5 } & SURF & $(4,4)$ & 0.0969 & 0.8993 \\
\hline \multirow{3}{*}{$\sigma_{\mathrm{n}}=50$} & Proposed & $(4,3)$ & 0.0873 & 0.8982 \\
\cline { 2 - 5 } & SIFT & $(5,4)$ & 0.1042 & 0.9078 \\
\cline { 2 - 5 } & SURF & $(4,4)$ & 0.0989 & 0.8969 \\
\hline \multirow{3}{*}{$\sigma_{\mathrm{n}}=100$} & Proposed & $(4,3)$ & 0.0873 & 0.8979 \\
\cline { 2 - 5 } & SIFT & Fail & Fail & Fail \\
\cline { 2 - 5 } & SURF & $(4,4)$ & 0.0935 & 0.8972 \\
\hline
\end{tabular}

Table 3. The overall estimated parameters for every already distorted image in the LIVE image database, which is further distorted by scaling factor 0.9 , rotation angle $0.1 \times 180 / \pi$ degrees, spatial shifts 4 pixels in the horizontal direction, 4 pixels in the vertical direction, and different noise levels.

\begin{tabular}{|c|c|c|c|c|}
\hline \multirow{2}{*}{$\begin{array}{c}\text { Noise } \\
\text { level }\end{array}$} & Method & Translation & Rotation & Scale \\
\cline { 2 - 5 } & Original & $\mathbf{( 4 , 4 )}$ & $\mathbf{0 . 1 0 0 0}$ & $\mathbf{0 . 9 0 0 0}$ \\
\hline \multirow{3}{*}{$\sigma_{\mathrm{n}}=0$} & Proposed & $(4,4)$ & 0.1047 & 0.8980 \\
\cline { 2 - 5 } & SIFT & $(4,4)$ & 0.1000 & 0.9002 \\
\cline { 2 - 5 } & SURF & $(4,5)$ & 0.0998 & 0.9010 \\
\hline \multirow{3}{*}{$\sigma_{\mathrm{n}}=50$} & Proposed & $(3,4)$ & 0.1047 & 0.8980 \\
\cline { 2 - 5 } & SIFT & $(4,4)$ & 0.0940 & 0.9037 \\
\cline { 2 - 5 } & SURF & $(9,-5)$ & 0.0813 & $1.45 \times 10^{13}$ \\
\hline \multirow{3}{*}{$\sigma_{\mathrm{n}}=100$} & Proposed & $(3,4)$ & 0.1047 & 0.8981 \\
\cline { 2 - 5 } & SIFT & $(5,3)$ & 0.0887 & 0.9067 \\
\cline { 2 - 5 } & SURF & $(23,-2)$ & 0.0117 & $2.2 \times 10^{14}$ \\
\hline
\end{tabular}




\section{Conclusions}

Image registration plays a very important role in a number of real-life applications. In this paper, we have proposed a new method to register reference and distorted images. Experimental results show that the proposed method performs very well for Barbara and Lena images, and with the LIVE Image Quality Assessment Database Release 2. In addition, our method works very well in noisy environments.

Even though the FFT and Radon transform have already been applied in image registration, this paper is different from them in the following ways. We calculate the scaling factor $a$ as the ratio of the means of the two input images. Since Gaussian white noise has zero mean, the scaling factor $a$ is invariant to the noise level. The rotation angle $\theta_{0}$ between the two images is estimated by using the Radon transform and the FFT-based fast cross-correlation. Because the Radon transform is robust to Gaussian noise, we can obtain the rotation angle $\theta_{0}$ accurately even if there is noise in the images. The global shifts between the two images are also robust to Gaussian noise according to [3].

\section{Acknowledgement}

The authors would like to thank the anonymous reviewers, the Associate Editor, and the Editor-in-Chief whose constructive comments and suggestions have improved the quality of this paper. This work was supported by Vantrix Corporation and by the National Sciences and Research Council of Canada (NSERC) under the collaborative research and development program (NSERC-CRD 326637-05).

\section{References}

[1] K. Keller, A. Averbuch and M. Israeli, "Pseudo polar-based estimation of large translations, rotations, and scalings in images," IEEE Transactions on Image Processing, vol. 14, no. 1, pp. 12-22, 2005.

[2] G. Wolberg and S. Zokai "Robust Image Registration Using Log-Polar Transform," Proceedings of the IEEE International Conference on Image Processing (ICIP), pp. 493-496, 2000.

[3] G. Varghese and Z. Wang, "Video denoising based on a spatiotemporal Gaussian scale mixture model," IEEE
Transactions on Circuits and Systems for Video Technology, vol. 20, no. 7, pp. 1032-1040, 2010.

[4] B. S. Reddy and B. N. Chatterji, "An FFT-based technique for translation, rotation and scale-invariant image registration," IEEE Transactions on Image Processing, vol. 5, no. 8, pp. 1266-1271, 1996.

[5] K. Jafari-Khouzani and H. Soltanian-Zadeh, "Radon transform orientation estimation for rotation invariant texture analysis," IEEE Transactions on Pattern Analysis and Machine Intelligence, vol. 27, no. 6, pp. 1004-1008, 2005.

[6] E. De Castro and C. Morandi, "Registration of translated and rotated images using finite Fourier transforms," IEEE Transactions on Pattern Analysis and Machine Intelligence, vol. PAMI-95, pp. 700-703, 1987.

[7] W. Wei, S. Wang, X. Zhang and Z. Tang, "Estimation of image rotation angle using interpolation-related spectral signatures with application to blind detection of image forgery," IEEE Transactions on Information Forensics and Security, vol. 5, no. 3, pp. 507-517, 2010.

[8] D. G. Lowe, "Distinctive Image Features from ScaleInvariant Keypoints," International Journal of Computer Vision, vol. 60, no. 2, pp. 91-110, 2004.

[9] H. Bay, A. Ess, T. Tuytelaars and L. Van Gool "SURF: Speeded Up Robust Features," Computer Vision and Image Understanding (CVIU), vol. 110, no. 3, pp. 346-359, 2008.

[10] Y. Pan, K. Li and M. Hamdi, "An improved constant-time algorithm for computing the radon and hough transforms on a reconfigurable mesh," IEEE Transactions on Systems, Man, and Cybernetics, Part A - Systems and Humans, vol. 29, no. 4, pp. 417-421, 1999.

[11] H. R. Sheikh, Z. Wang, L. Cormack and A. C. Bovik, "LIVE image quality assessment database release 2," http://live.ece.utexas.edu/research/quality.

[12] D. Nikolic, R. C. Muresan, W., Feng and W. Singer, "Scaled correlation analysis: a better way to compute a crosscorrelogram," European Journal of Neuroscience, pp. 1-21, 2012.

[13] V. I. Sobolev, "Convolution of functions," in Michiel Hazewinkel, Encyclopedia of Mathematics, Springer, ISBN 978-1-55608-010-4.

[14] A. Averbuch, R. R. Coifman, D. L. Donoho, M. Israeli, and Y. Shkolnisky. A framework for discrete integral transformations I - the pseudo-polar Fourier transform. SIAM Journal on Scientific Computing, 30(2): 764-784, 2008.

[15] C. Mythili and V. Kavitha, "Efficient technique for color image noise reduction," The Research Bulletin of Jordan ACM, vol. II (III), pp. 41-44, 2011. 


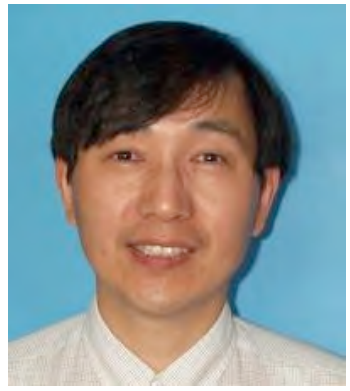

Guangyi Chen holds a B.Sc. in Applied Mathematics, an M.Sc. in Computing Mathematics, an M.Sc. in Computer Science, and a Ph.D. in Computer Science. During his graduate and postdoctoral studies, he was awarded a Visiting Fellowship in Canadian Government Laboratories, an NSERC postdoctoral fellowship, a Canadian Space Agency Postdoctoral Fellowship Supplement, an NSERC PGS B Fellowship, an FCAR Scholarship, the J.W. McConnell Memorial Graduate Fellowship, and the Concordia University External Award Holder Doctoral Fellowship. He is currently an Editor for the International Journal of Applied Mathematics and Statistics. His research interests include pattern recognition, signal/image/video processing, machine learning, artificial intelligence, and scientific computing.

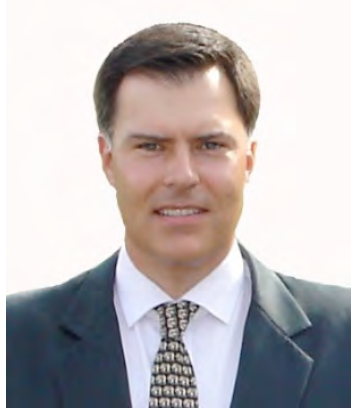

Stéphane Coulombe is a Professor in the Software and IT Engineering Department of the École de technologie supérieure (ÉTS) (part of the Université du Québec network), a leading engineering school located in Montreal. He received his B.Eng. in electrical engineering from the École Polytechnique de Montréal, Canada, in 1991, and his Ph.D. in telecommunications (image processing) from INRS-Telecommunications, Montreal, in 1996. From 1997 to 1999, he worked for Nortel's Wireless Network Group in Montreal, and from 1999 to 2004, he worked at the Nokia Research Center in Dallas, Texas, as Senior Engineer, and as Program Manager in the Audiovisual Systems Laboratory. At Nokia, he led research and development activities related to media adaptation and transcoding. In 2004, he joined ÉTS, where he currently leads research and development activities related to video processing and systems, media adaptation, and transcoding. Since 2009, Professor Coulombe has held the Vantrix Industrial Research Chair in Video Optimization. 


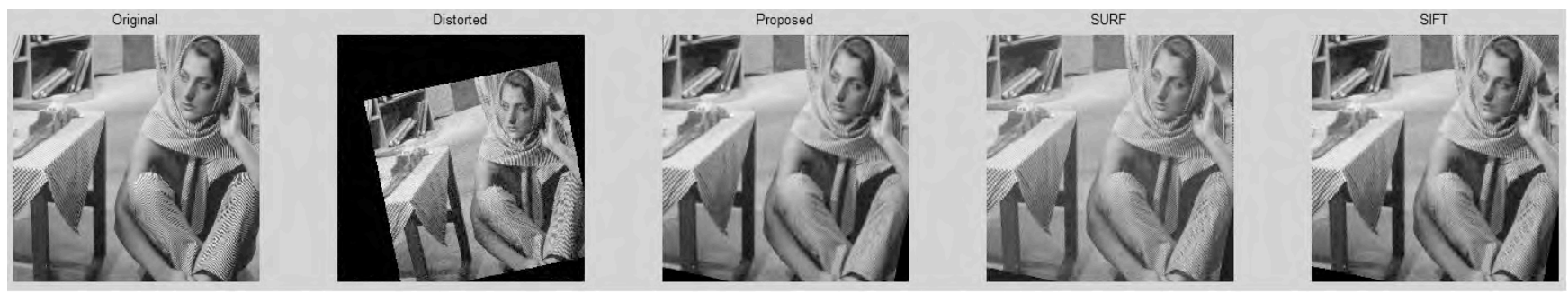

Fig. 1. The original image, its distorted version (scale $=0.8$, rotation $=0.2$ radian, translation $=(40,40)$, the noise standard deviation $\sigma_{\mathrm{n}}=10$ ), and the images registered using the proposed method, SURF, and SIFT, respectively. It can be seen that all three methods work well for such big spatial shifts $(40,40)$.
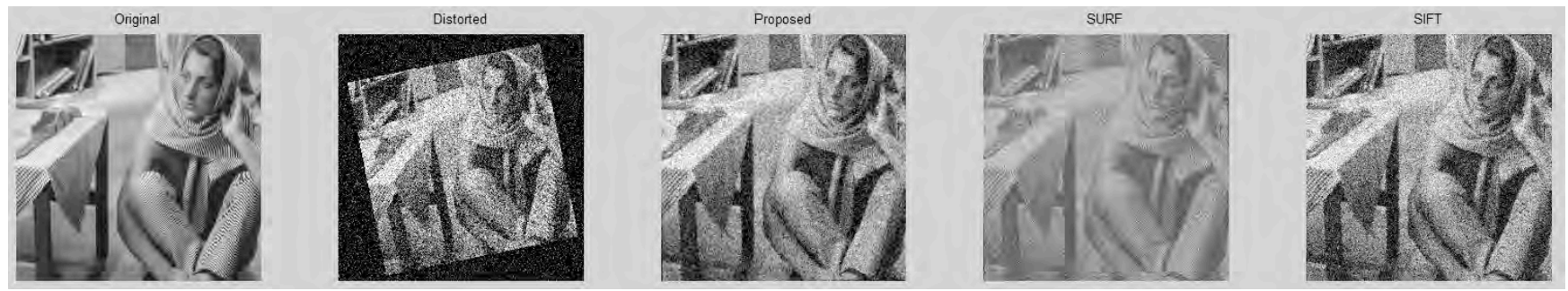

Fig. 2. The original image, its distorted version (scale $=0.8$, rotation $=0.2$ radian, translation $=(4,4)$, the noise standard deviation $\sigma_{\mathrm{n}}=50$ ), and the images registered using the proposed method, SURF, and SIFT, respectively.
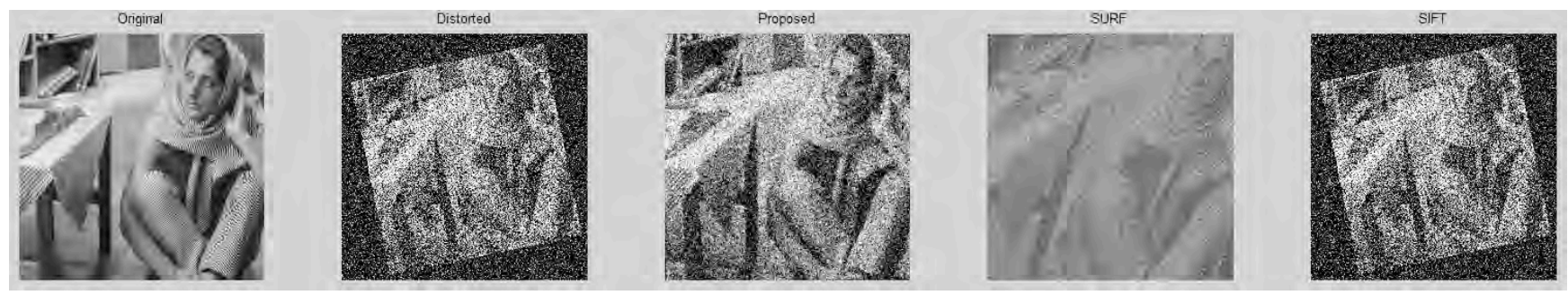

Fig. 3. The original image, its distorted version (scale $=0.8$, rotation $=0.2$ radian, translation $=(4,4)$, the noise standard deviation $\sigma_{\mathrm{n}}=100$ ), and the images registered using the proposed method, SURF, and SIFT, respectively. It can be seen that both SURF and SIFT fail, but our proposed method works well in this case.
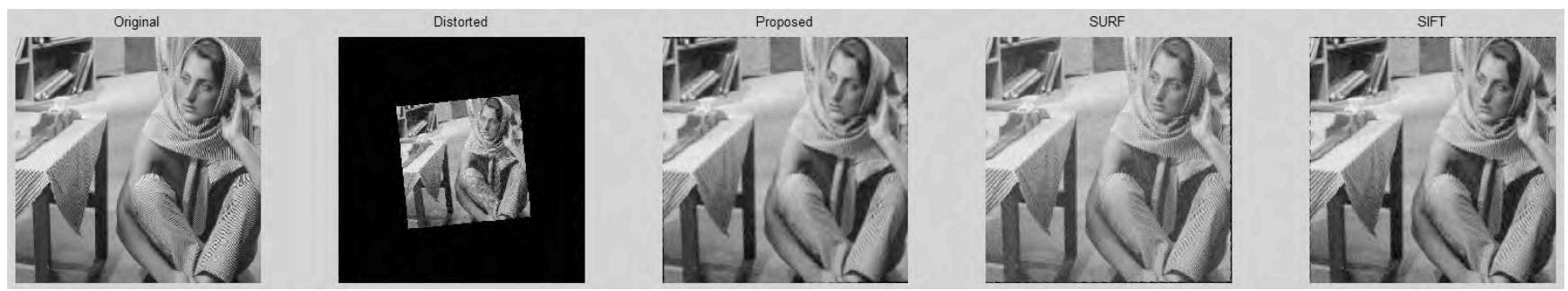

Fig. 4. The original image, its distorted version (scale $=0.5$, rotation $=0.2$ radian, translation $=(4,4)$, the noise standard deviation $\sigma_{\mathrm{n}}=10$ ), and the images registered using the proposed method, SURF, and SIFT, respectively. With scaling factor of 0.5 , all three methods perform well.

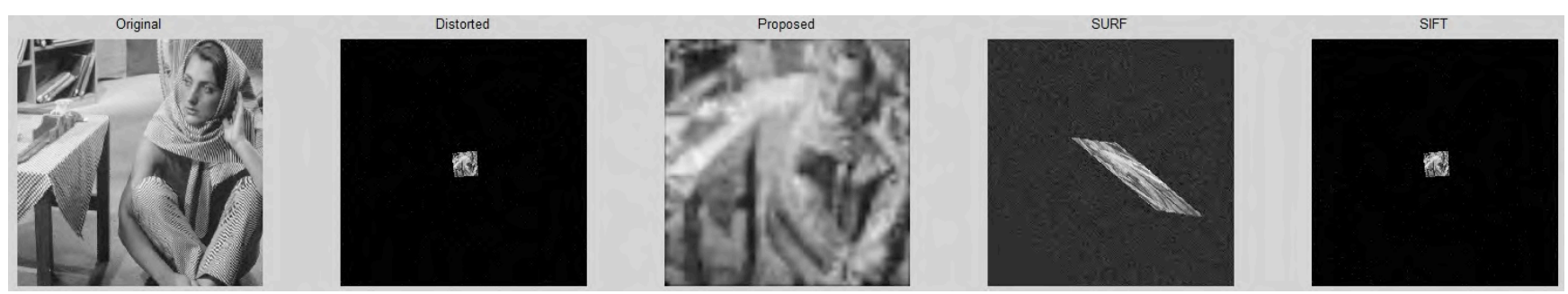

Fig. 5. The original image, its distorted version (scale $=0.1$, rotation $=0.2$ radian, translation $=(4,4)$, the noise standard deviation $\sigma_{\mathrm{n}}=10$ ), and the images registered using the proposed method, SURF, and SIFT, respectively. With scaling factor of 0.1 , our proposed method performs very well. However, both SURF and SIFT fail. 